\title{
Evaluation of XML Schema Support in Knowledge Management
}

\author{
Boštjan ŠUMAK ${ }^{\mathrm{a} 1}$, Marjan HERIČKO ${ }^{\mathrm{a} 2}$ and Maja PUŠNIK ${ }^{\mathrm{a}, 3}$ \\ ${ }^{a}$ University of Maribor, Faculty of Electrical Engineering and Computer Science
}

\begin{abstract}
Well organized data contributes extensively to the classification possibilities and quality of Knowledge Management. XML schemas play an important role in data organization activities, and provide basic foundations for companies and organizations dealing with large amounts of data. In times where knowledge represents the greatest advantage in a competitive economy and is relatively simple to find through different web providers, the quality of internal data structures and efficient management of a company's valuable information is of the utmost importance. XML schemas are one of the mechanisms that can provide a data organization system in a qualitative manner, and efficient knowledge management as soon as data have been defined or accumulated. A good XML schema support is a way to increase the competitiveness of an organization by ensuring structured data quality and simplifying the Knowledge Management process.
\end{abstract}

Keywords. Information Management, Data Management, Knowledge Management, XML schema, XML document, Data quality

\section{Introduction}

Knowledge is a product of well-developed data and, by definition, Knowledge Management $(\mathrm{KM})$ is an efficient handling of information and resources within an organization. It is the process of capturing, developing, sharing, and effectively using organizational knowledge [1] by making the best out of given data. It's an interdisciplinary field, an intersection of Computer Science and management, addressing benefits in organizational aspects of business sectors, however, relying greatly on Computer and Information Science. KM has been around since the mid-1990s, and its attempts to collect, store, categorize, mine, and process data into knowledge [31], correspond to Big Data challenges (capturing data, data storage, data analysis, search, sharing, transfer, visualization, as well as querying, updating, information privacy and data source), in both cases dealing with large size data.

Managing large size data or knowledge within an organization is unimaginable without the use of advanced information systems, while the design and implementation of such systems demands great organization, as well as presents several technical

${ }^{1}$ Boštjan Šumak, University of Maribor, Faculty of Electrical Engineering and Computer Science, Koroška cesta 46, SI-2000 Maribor, Slovenia; E-mail: bostjan.sumak@um.si.

2 Marjan Heričko, University of Maribor, Faculty of Electrical Engineering and Computer Science, Koroška cesta 46, SI-2000 Maribor, Slovenia; E-mail: marjan.hericko@um.si.

${ }^{3}$ Maja Pušnik, Corresponding author, University of Maribor, Faculty of Electrical Engineering and Computer Science, Koroška cesta 46, SI-2000 Maribor, Slovenia; E-mail: maja.pusnik@um.si. 
challenges. Another closely tied discipline is Knowledge Engineering, which refers to all technical, scientific and social aspects involved in building, maintaining and using knowledge-based systems, which is vital for enterprise interoperability and networking.

The paper focuses on advanced information and knowledge based systems, supporting KM. Furthermore, it is focusing specifically on the data part of IT systems, containing the potential for creating knowledge. The data can originate in several forms, where common denominators are often XML documents and XML schemas, defining the structure of XML data. The quality of the data structure is a precondition for the knowledge based system, and the basis for many other disciplines, such as Machine Learning, Data Mining, and others. Difficulties and the importance in building qualitative data sets are addressed in this paper, focusing on XML schemas as the de facto Standard for data interchange [2], and an important part of Information Solutions, considered a prerequisite to Knowledge Management.

In times of e-business, data are exchanged based on XML documents, a mark-up language that defines a set of rules for encoding documents in a format that is both human-readable as well as machine-readable. Several other formats (MS Office, Open office), can be translated easily into XML files, which provide users several advantages, information and knowledge extraction being only some of them. Each XML document is defined by an XML schema (https://www.w3.org/XML/Schema), the focus technology of this paper. The structure and contents of XML schema documents have a significant influence on the value of data, which influences data quality in general indirectly, presenting a challenge in the first levels of information and KM. A good XML schema leads to a higher efficiency of data usage, extraction and organization, as well as simplification of Information Solutions, simplified maintenance and, therefore, higher quality of data, information and knowledge. The paper focuses on measuring the quality of XML schemas, and addresses it from a structural and contents/data perspective, presenting data as the heart of today's economy [3], as well as the fundament of KM.

Companies use different databases, often including some sort of XML contents, due to the e-oriented business world, suitable for web presentation of data in various domains and digital libraries, which allow users to find anticipated search items [4]. Based on empirical research of 200 case studies of XML schema usage [5] (from various humanistic, natural and technical domains), XML schemas are often built in a manner merely satisfying the minimum requirements of syntactic correctness and contents sufficiency (the effort, however, varies between the previously mentioned domains). Often this is the problem with generated XML schemas or an inappropriate (simplified) approach to XML schema building by non-experts. Difficulties occur when extending, reforming or reusing XML schemas.

Existing metrics for quality evaluation only address the problem partially; they base their metrics on existing solutions known in Software Engineering, and do not address the problem of an objective quality evaluation. Dynamic creation and adaptation of XML schemas presents an additional research challenge that requires the use of new approaches and solutions. Additionally, while the structure rules are mostly acquired, a good data organization is often neglected, and often insufficient, especially in the case of large size data. For example, in the case of Big Data, where volume, velocity, and variety are emphasized (in addition to veracity, variability, visualization and value of the gathered data), the quality of data organization is crucial, suitably addressing challenges specific to big data [25]. As the authors in [25] point out: Big Data is simply a lot of data, and with IT support the user should be able to import and process data based on specific criteria defined by the user (in the form of an XML schema or otherwise). The ability to 
handle different kinds of data sources in a systematic and controlled manner, with the goal of generating suitable information for organizing data, would provide the most value to the user. XML schemas and Big Data combined also provide possibilities to evaluate not only the quality of data, but also enable a quantitative analysis of the attitudes, intentions and motivation of (XML schema) users, possibly providing insight about descriptive and predictive user behavior. In this paper, the quality of XML schemas is evaluated on a structural level of 200 examined examples, although an additional quantitative analysis (statistical modeling for good data structure practices' identification) can improve XML schema quality immensely, KM support can also reduce the risk of misunderstanding data. Based on [25] and [32], the hierarchy of data, information, knowledge and how it connects to XML technologies, is presented in Figure 1.

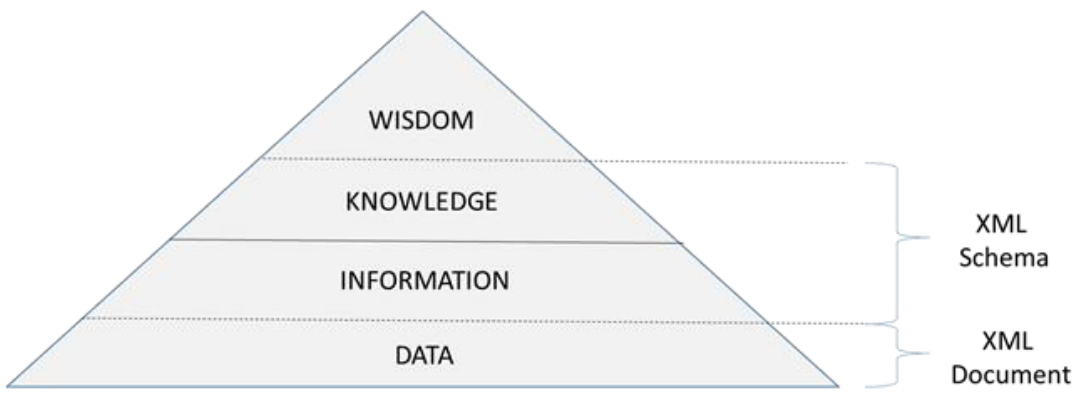

Figure 1 The hierarchy of data, information, knowledge and wisdom [11], connected to XML technologies

The aim of this paper is to focus on KM needs and ensure evaluation of XML data quality in business documents, supporting a better quality of data organization. Theoretical knowledge was tested on an expanded set of test data of already established XML schemas (for business documents) in various organizations, with the help of composite metrics. The paper's main research question investigates, how XML schema characteristics impact the quality of data, and influence Knowledge Management possibilities. XML schema quality aspects were defined to provide an answer. A set of metrics for assessing different quality aspects of XML schemes was developed, and results were combined with the XML Schema Quality Index, a tool for reporting the data quality of XML schemas [5]. In the following sections, those aspects are presented, indicating that quality of XML schemas address KM quality as well.

The structure of the paper is as follows: After an introduction to the problem (Section 1), the paper presents related work on XML schema quality research (Section 2), and establishment of the Knowledge Management connection with the XML schemas is provided (Section 3). Section 4 focuses on quality aspects, and results of the analysis are provided in Section 5. The conclusion (Section 6) holds a summary of results and future research plans are given.

\section{Related work}

Several scientific and professional papers are addressing the quality of XML schemas in software applications in general within the time scope of the last 15 years. There have 
been few attempts to evaluate and measure XML schemas, however, the number of evaluated XML schemas was low, and the quality aspect of those XML schemas was mostly one-dimensional, focusing only on complexity. Several metrics are summed by Zhang in [8], and significantly related work was done by [6], [7] and [8], where attempts were made to measure XML schemas and software in general. Finding suitable metrics for XML technologies was addressed in other papers, althoughtheir backgrounds, being mainly software metrics, are not applicable to all data aspects of XML schema quality measurements, the main focus in this paper. The authors in [9] address metrics for evaluation of XML schema structure complexity, pointing out entropy. The proposed metric is evaluated empirically, based on a case study. While applying the metric, the authors do not include a variety of preferences for XML schemas based on their purpose or data domain. The authors in [10] share the approach to measuring XML schema quality; however, they consider structural aspect, as well as that of contents. Their metric enables user modification of quality measurement settings. In our paper, we take a step forward and include several other aspects of XML schema quality, although not providing user modification possibilities.

Improving XML schema quality is the focus in [11], addressing the problem of changing existing XML documents (according to XML schema changes). To present the problems, each change has an assigned cost and preference weight. A scalar measurement is provided for each XML schema quality aspect, as proposed and developed further in this paper. Assigning properties and rules in XML schemas is addressed in [12], focusing on information system integration and the role of XML schemas in the integration process. Furthermore, the evaluation of standard quality in [12] is a simplified version of the evaluation proposed in our paper. The complexity aspect is addressed in [13], based on the internal structure of XML schemas, and providing validation variables and a measurement method for calculating complexity. The above mentioned researches depict several variables important for XML schema quality evaluation and measurement, although they stay mostly on the data level, and do not address the quality aspects affecting the Knowledge Management based on XML schema defined documents. The main focus on the data level quality, respectively, data bases and XML schemas, is evident in papers [20] and [21], enforcing the important connection of XML schemas and data sources. According to [2], the integration of heterogeneous data sources has become a central problem of modern computing, especially in domains where data currently reside in many different formats. In addition to a relational database, XML data can be stored in an object-oriented database, or in a semi-structured system, providing more possibilities for KM activities.

A lot of effort and application of XML related technologies was also addressed within the EJC conference on all levels of IT development (from concepts, models, and data bases to a higher logic layer and user interfaces, applying XML technologies as a format for data extraction). The usefulness and wide application of XML documents and schemas is addressed in [24], and the authors in [22] presented the widespread usability of XML, by describing a conceptual model for XML, dividing the modeling process into conceptual and structural levels, using XML documents, as well as XML schemas. The author in [23] developed a framework for modeling and implementing layered database applications, defining a database model for each of the layers and a facility for exchange of data throughout different layers in a coherent form, using XML as a hierarchical modeling language for internal, conceptual and external schemas. The connection of (XML) data and knowledge is addressed clearly in [25], while XML application at all levels of development of IT solutions is presented in [26], where the output of a 
developed algorithm is produced as an XML file. XML is clearly infiltrated in all levels of IT architecture, and the authors in [27] address the use of XML in modeling (used for communication between interest groups and inside development teams), revealing different modeling cultures and emphasizing the significance of data planning [28] [29] [30] and the importance of XML supporting technologies in Knowledge Management.

\section{Knowledge Management and XML schemas}

The amount of available knowledge has grown in the past years, bringing challenges for controlling and managing it [14]. Well collected and organized data are easily summarized and analyzed, providing possibilities of synthesizing information into knowledge, enabling several advantages to organizations. Efficient classification of data, for example, important for various fields, such as Libraries Science [15], is well supported by XML schemas through different restriction building blocks. In addition, a suitable use of XML schema building blocks can largely support multilingual data, often necessary in the information globalization process [16]. The low-level data collection process is a fundament for higher knowledge creation levels, indicating the importance of data quality, which is often received and stored in XML documents. Figure 2 includes basic transformation levels from data to knowledge, and connects them with XML schema building from basic structures to advanced XML schema use, indicating the knowledge about data. In the last years, knowledge has become an important wealth indicator, and KM has become a critical factor for organizations and an important tool for international and local competition. The goal of $\mathrm{KM}$ is to make organization, classification and validation work more effective; it's not just about the knowledge itself, but about how to use and spread the knowledge in the most effective and wide way. In order to be valuable, knowledge must be shared [14], while (quality) XML schemas provide support within the KM process.

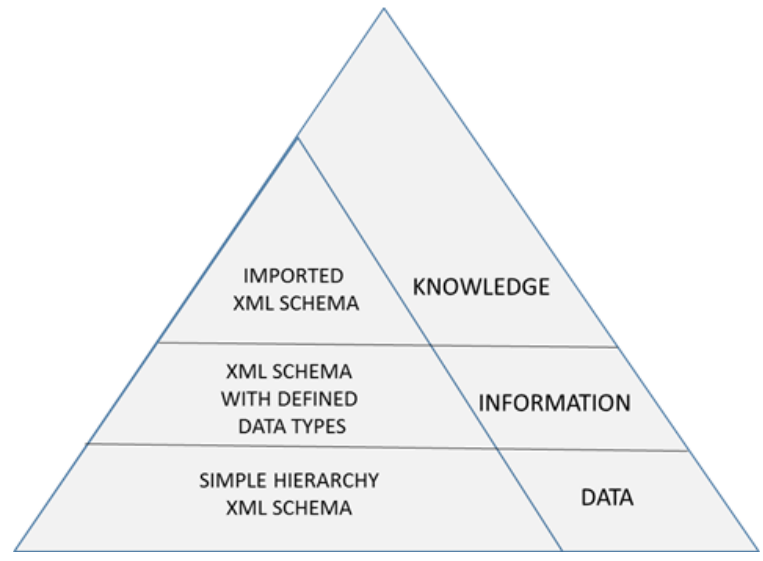

Figure 2. Data, information and Knowledge Management [1]

$\mathrm{KM}$ is the process of capturing, developing, sharing, and using organizational knowledge effectively [17]. On the level of using XML schemas, organization and classification are the most important aspects. The Knowledge Management methodology has been 
designed to ensure a proper, comprehensive, systematic and consistent approach, and is, in many ways, coherent with XML schema development (respectively quality levels). It ensures proper attention is given to the critical success factors and quality aspects in implementing Knowledge Management programs successfully [18]. Several process steps are depicted in Figure 3, connected with XML schema quality levels. The XML schema building process consists of the following levels:

- A simple hierarchy XML schema includes only element and attribute sequence and cardinality definitions,

- Local data type definitions add information to each element or attribute about its existing or newly defined data type,

- Global data type definitions provide globally defined building blocks for increased reuse, especially in cases of external XML schema use,

- The Quality Index indicates how advanced and controlled defined XML schemas actually are.

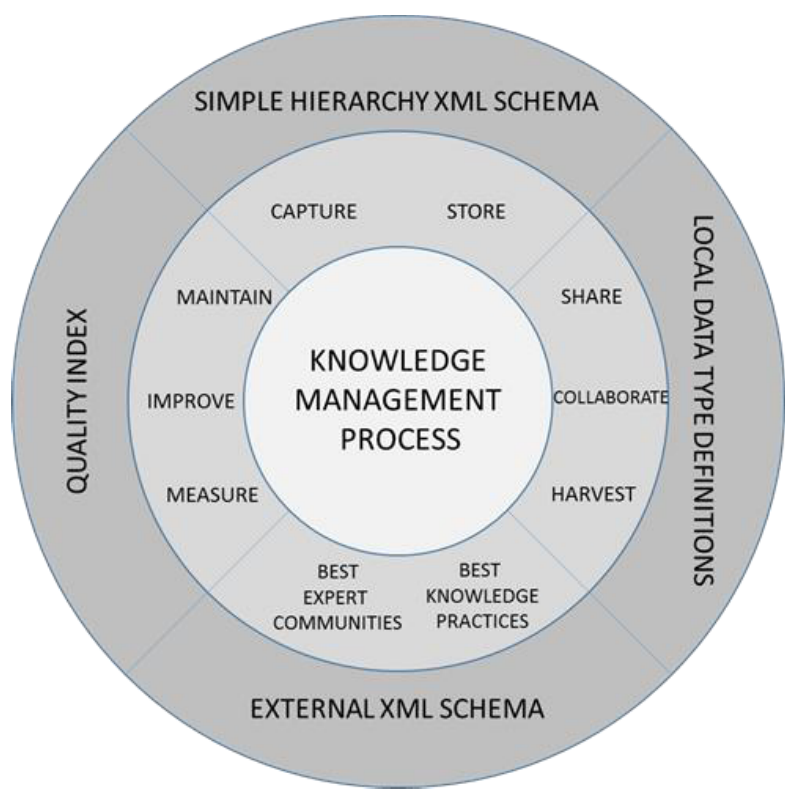

Figure 3. Knowledge Management process and XML schemas [18]

A competitive advantage of organizations is gaining all possible knowledge to assimilate and learn faster. The need for Knowledge Management is infiltrated in several organizational frameworks using XML documents as well. Knowledge Management in the software industry deals with the problem of having specialists of a narrow sector, not always in tune with other knowledge intensive branches using their products [19]. Therefore, Data Refinement and Information Extraction are crucial for understanding and gathering knowledge. A quality XML schema simplifies this task, benefiting Knowledge Management in organizations, as well as providing a metric, estimating the quality of existing XML schemas. The main XML schema building blocks, influencing its quality, will be indicated, providing a guideline to build more adapted XML schemas, suitable for data definition in the first level of the Knowledge Management process. 


\section{Measuring the quality of data in XML schemas}

XML schemas define the structure and content of all elements and attributes, storing valuable organizational data in XML documents, which is gaining in popularity [4]. It provides a number of features that make it suitable for web based application. Its role is definition of all elements and attributes, organization of the hierarchy, sequence and cardinality of data elements. Each element or attribute is defined (its structure and contents) by a data type or integrated building blocks. Covering a wide range of data demands, organized and well-formed exchange of documents is essential in different domains of e-business (e-Health, e-Library, e-Government, etc.), web applications as well as data storage. XML schemas must, therefore, be extendable and flexible, suitable for future changes, and have a rich set of building blocks. This is especially important when integrating data from different XML schemas.

An XML schema is a model defining document structure and enabling a creation of an agreement regarding structure and contents, security supported via XML encryption. It enables a simplified creation of complex content models, focused on reuse of elements and attributes, as well as data types, storing valuable data. One of most significant advantages of XML schemas is data type support, simplifying the document contents' description, validation of data correctness, defining data restrictions and data patterns. XML schemas are extendible, and can be reused within other XML schemas. Within one XML document there is a possibility of holding several XML schemas (by including or importing a building block), spreading a set of possible types and/or elements. A wellshaped XML document is a document adapted to all XML syntactical rules, as are XML declaration, one root element, suitable tag names, case- sensitivity, end tags, the hierarchy rules and attribute values within quotes. Although an XML document is well formed, errors can still exist, having serious consequences on data storage and system well-being. The problem is addressed by a suitable approach to building XML schemas, where good practices already exist.

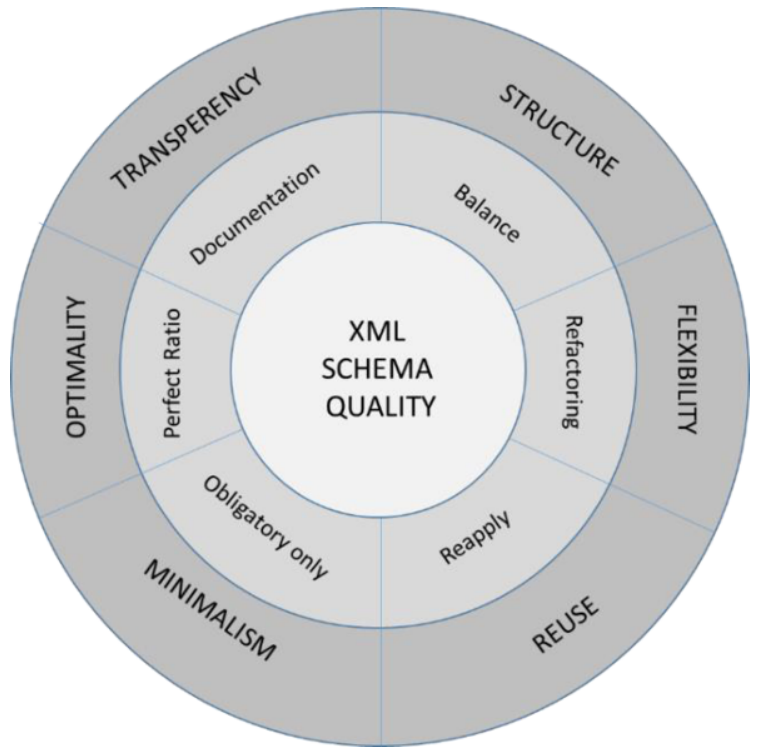

Figure 4. XML schema quality aspects [19] 
Literature review results of the XML schema quality measurements field provided several metrics, linked historically to approaches and measurements methods in Program Engineering, focusing mostly on XML schemas` structure and complexity. Based on the definition of structure metrics, the paper includes other aspects of XML schema quality, also important for advanced Knowledge Management, summarized based on ISO/IEC 9126, theory of decision models, and other papers. While choosing metrics with which we wish to improve XML schema quality, the paper includes parameters, effecting XML schema improvement (Figure 4):

Figure 4 presents a set of parameters organized in 6 aspects. A composite metric is combining all six aspects equally (structure, clarity, optimality, minimalizm, re-use, flexibility), presenting a general Quality Index, and defining a joint grade of quality.

\section{Results}

Two types of XML schemas were included: (1) XML schemas, designed through a standardization process of larger independent organizations (W3C, OASIS), and (2) XML schemas, defined within different companies and organizations for needs of ebusiness and Knowledge Management. All schemas were analyzed, and measurable elements were extracted based on a database of 200 XML schemas found in the search process. The analysis was semi-manual with help of a tool developed in the Institute of Informatics, University of Maribor.

Table 1. Overview of positive and negative variables

\begin{tabular}{ll}
\hline \multicolumn{1}{c}{ Predictor } & Beta value \\
\hline Number of included XML schemas &, 164 \\
XML schema type &,- 384 \\
Number of local elements &,- 017 \\
Number of global complex elements &, 308 \\
Number of global simple elements &, 145 \\
Lines of code &,- 012 \\
Number of attribute groups &, 133 \\
Number of annotations &, 130 \\
Number of derived data types &, 047 \\
\hline
\end{tabular}

Analysis of data was conducted by regression analysis with the help of a standard linear regression. Table 1 presents variables with significant impact on the quality of XML schemas. The Beta indicator presents a value (positive or negative), indicating the addition or deduction of an XML schema quality in case an additional variable occurs in an XML schema. For example, with each additional external XML schema (Number of included XML schemas), the quality of an XML schema goes higher by 0.1645 on a scale from 0 to 1 , indicating that, in fact, the XML schema`s characteristics (measured parameters) impact it's quality, and, furthermore, the possibilities of qualitative Knowledge Management

Overall findings about XML schema use produced the following results [33]: The structure of XML schemas influences the quality of data application, although different domains require different XML schema types: Economy related fields typically require a very non-flexible and strict structure, while technical and humanistic domains are much less strict. The most flexible XML schema structure is required in Natural Science domains, where possibly the pool of data varies the most. The general conclusion is that 
no XML schema type can be applied on all domains and needs, as well as that the understanding of what makes a useful XML schema varies. Also, not all aspects can be equality satisfied, although there are some good practices which can be used as general guidelines, presented in Table 2 (compared to average XML schemas):

Table 2. Good and bad characteristics of general XML schemas.

\begin{tabular}{|c|c|c|}
\hline & $\begin{array}{c}\text { Standardized properties of the } \\
\text { average XML schema }\end{array}$ & Good XML Schema Properties \\
\hline $\begin{array}{l}\text { Use of external } \\
\text { schemes }\end{array}$ & $\begin{array}{l}0.8 \text { use external schema per XML } \\
\text { schema (import or include) }\end{array}$ & $\begin{array}{l}\text { Minimum usage ( } 1 \text { or } 2) \text {, suitable for } \\
\text { separating data type definitions from } \\
\text { element and attribute definitions. }\end{array}$ \\
\hline Use of elements & $\begin{array}{l}3 \times \text { more local than global } \\
2 x \text { more complex than simple }\end{array}$ & $\begin{array}{l}\text { Global 1, other local. It uses more in } \\
\text { exceptional cases when an XML schema } \\
\text { defines multiple XML documents. }\end{array}$ \\
\hline Use of attributes & $\begin{array}{l}\text { Typically local attributes, } 0.6 \text { attributes } \\
\text { per element, }\end{array}$ & Attributes minimum (0.05 per item). \\
\hline Use of groups & 4.4 groups per XML schema. & Not recommended \\
\hline Use of data types & 2.3 more simple data types & $\begin{array}{l}\text { Almost as many global data types as } \\
\text { there are elements and attributes. Simple } \\
\text { are less than complex; a better complex } \\
\text { element with simple content that makes } \\
\text { it easier to modify the XML schema in } \\
\text { the future. }\end{array}$ \\
\hline Documentation & 1 annotation per complete scheme & $\begin{array}{l}\text { Annotation affects the quality of XML } \\
\text { schemas, but minimal documentation is } \\
\text { sufficient to improve it. }\end{array}$ \\
\hline Limits & $\begin{array}{l}1.3 \text { restrictions per individual building } \\
\text { block. }\end{array}$ & Each block should be restricted. \\
\hline
\end{tabular}

\section{Conclusion}

The paper addresses Knowledge Management issues through XML schemas, their characteristics, influence and contribution to knowledge organization and classification, focused on assessing the quality of XML schemas. The literature review revealed the importance of XML schemas within XML technologies, and their importance in integration of Information Systems and Knowledge Management. In the paper we have analyzed the demands of quality in XML schemas and the meaning of XML schemas in Knowledge Management organizations.

We have listed the most important building blocks for quality evaluation by experimenting on 200 XML schemas of different origin. XML schemas were analyzed with regression analysis to establish which characteristics (building blocks) influence the quality of XML schemas the most, indicating critical quality aspects for future building of XML schemas.

Six aspects of quality were defined, combined into one value: The Quality Index. The quality aspects included (1) Structure, (2) Transparency, (3) Optimality, (4) Minimalizm, (5) Reuse and (6) Integrability. All aspects were measured separately; a regression analysis was conducted, discovering nine variables (positively or negatively) influencing the final XML schema quality estimation significantly. Results exposed the following parameters as the most significant: The number of included external XML schemas, XML schema type, three variables connected to element use, number of attribute groups, lines of code, annotation usage and number of derived data types. All 
significant variables are, in different forms, included in Metrics, addressing specific aspects of XML schema quality estimation, providing an answer to the research question: Do XML schema characteristics impact the quality of data, influencing Knowledge Management possibilities? A more detailed impact of XML schema quality on Knowledge Management is yet to be confirmed empirically in the future work. The results in this paper provide possibilities for further scientific and applicative research in the field of XML Schema Quality, as well as in the field of XML Data Impact on Knowledge Management. Additional note: Based on our research, 30\% of XML schemas were not built properly and need adaptation, mostly in the fields of structure and transparency, therefore not providing enough support for Knowledge Management

The research within the paper also has limitations due to human error when analyzing XML schemas. To confirm the validity of the results, the research should be repeated. The future work will address the Knowledge Management efficiency connected with XML schema quality in more detail, as well as the influence of contents (data) on XML schema quality aspects. To address these aspects efficiently, new refined metrics will be introduced, tailored according to Knowledge Management specific needs. An additional future study will include measurement of higher architectural levels of IT solutions, and how foundational XML schemas correlate with software quality, perceived usefulness of the end product and user experience of creating, sharing, using and managing the knowledge and information to achieve organizational objectives by making the best use of data and knowledge. The possibilities will also be examined of integrating Big Data concepts.

\section{References}

[1] T.H. Davenport, Saving IT's Soul: Human Centered Information Management, Harvard Business Review 72 (1994), 119-131.

[2] S.K. Madria, XML schema and data management, Data \& Knowledge Engineering. 52 (2005), 181-183.

[3] A, Uden, Developing Data Analytics to Improve Services in a mechanical Engineering Company, Knowledge. Management. Organization (2014).

[4] S. Goswami, C. Kundu, XML based advanced distributed database: Implemented on library system, International Journal of Information Management 33 (2013), 28-31.

[5] M. Pusnik, M. Hericko, Z. Budimac, B. Sumak, XML schema metrics for quality evaluation, Computer Science and Information Systems 11 (2014), 1271-1290.

[6] B. Sumak, M. Hercko, M. Pusnik, Towards a Framework for Quality XML Schema Evaluation Keywords, Information Technology Interfaces (2007), 783-788.

[7] K.M.A.S.C. Yue, Analysis and Metrics of XML Schema, Proceedings of the International Conference on Software Engineering Research and Practice and Conference on Programming Languages and Compilers (2004), 538-544.

[8] V. L. Narasimhan, B. Hendradjaya, Detailed theoretical considerations for a suite of metrics for integration of software components, Advances in Systems, Computing Sciences and Software Engineering 177 (2006), 257-264.

[9] D. Basci, S. Misra, Entropy as a Measure of Quality of XML Schema Document, The International Arab Journal of Information Technology 8 (2010), 16-24.

[10] E. Pardede, T. Gaur, On the Development of a User-Defined Quality Measurement Tool for XML Documents, Information Systems Development, Springer New York (2011), 213-221.

[11] Z. Tan, L. Zhang, Improving XML Data Quality with Functional Dependencies, Database Systems for Advanced Applications (2011), 450-465.

[12] K.C. Morris, A framework for XML schema naming and design rules development tools, Computer Standard Interfaces 32 (2010), 179-184.

[13] D. Basci, S. Misra, Measuring and evaluating a design complexity metric for XML schema documents, Journal of Information Science and Engineering 25 (2009), 1405-1425.

[14] R. Suurla, Avauksia tietämyksen hallintaan, Helsinki, Oy Edita Ab. (2001), 190. 
[15] U.A. Mokhtar, M.Y. Zawiyah, The requirement for developing functional records classification, International Journal of Information Management 35 (2015), 403-407.

[16] J. Zhang, Y. Sun, A.J. Jara, Towards semantically linked multilingual corpus, International Journal of Information Management 35 (2015), 387-395.

[17] K. Management, E. Factors, Knowledge Management Enabler Factors and Competitive Advantage: An Empirical Study in Pharmaceutical Industry in Andhra Pradesh, India, The International Journal Of Business \& Management 2 (2014), 104-108.

[18] KM Consulting Methodology Overview, http://www.knowledge-management-online.com/KMconsulting-method-overview.html. (Accessed March $1^{\text {st }} 2020$ ).

[19] Finland \& Knowledge Management, https://knowledgeandmanagement.wordpress.com/finlandknowledge-management/. (Accessed March $1^{\text {st }}$ 2020).

[20] E. Gallinucci, M. Golfarelli, S. Rizzi, Schema profiling of document-oriented databases, Information Systems 75 (2018), 13-25.

[21] Z. Brahmia, H. Hamrouni, R. Bouaziz, XML data manipulation in conventional and temporal XML databases: A survey, Computer Science Review 36 (2020).

[22] M. Necasky, J. Pokorny, Conceptual Modeling of IS-A hierarchies for XML. In: Proceedings of the 18th European Japanese ' Conference on Information Modelling and Knowledge Bases (2008), 65-84.

[23] B. Thalheim, Model suites for multi-layered database modelling. In: Proceedings of the 2010 conference on Information Modelling and Knowledge Bases XXI (2010), 116-134.

[24] S. Hartmann, S. Link, T. Trinh, Boolean Constraints for XML Modeling. In: Frontiers in Artificial Intelligence and Applications (2008), 85-104.

[25] P. Sillberg, Toward Manageable Data Sources. In: Proceedings of the 28th International Conference on Information Modelling and Knowledge Bases (2018), 485-494.

[26] M. Duží, M. Fait, Type checking algorithm for the TIL-Script language. In: Proceedings of the 28th International Conference on Information Modelling and Knowledge Bases (2018), 201-219.

[27] H. Jaakkola, B. Thalheim, Modelling Cultures. In: Proceedings of the 28th International Conference on Information Modelling and Knowledge Bases (2018), 33 - 52.

[28] G. Polančič, B. Šumak, M. Pušnik, A case-based analysis of process modeling for public administration system design. Information modelling and knowledge bases XXXI. Amsterdam: IOS Press (2020), 92104.

[29] B. Thalheim, H. Jaakkola, Models as Programs: The Envisioned and Principal Key to True Fifth Generation Programming. Information modelling and knowledge bases XXXI (2020).

[30] J. Musto, A. Dahanayake, Improving data quality, privacy and provenance in citizen science applications. Information modelling and knowledge bases XXXI (2020).

[31] S.Hijazi, Big Data and Knowledge Management: A Possible Course to Combine them Together. In: ASCUE Proceedings (2017).

[32] J. Rowley, The wisdom hierarchy: representations of the DIKW hierarchy. Journal of Information Science, 33 (2007).

[33] M. Pušnik, Quality evaluation of domain specific XML schemas. Doctoral dissertation. University of Maribor, Faculty of Electrical Engineering and Computer Science; 2014. 